\title{
TITLE:
}

\section{$<$ Note> Preliminary Observations of Hand-Clasp Grooming by Chimpanzees at Bulindi, Uganda}

\section{$\operatorname{AUTHOR}(S)$ :}

McLennan, Matthew R.

\section{CITATION:}

McLennan, Matthew R.. < Note> Preliminary Observations of Hand-Clasp Grooming by Chimpanzees at Bulindi, Uganda. Pan Africa News 2011, 18(2): $18-20$

ISSUE DATE:

2011-12

URL:

http://hdl.handle.net/2433/152162

\section{RIGHT:}

Copyright (C) Pan Africa News. 
leaf-folding at Bossou? There are several differences ${ }^{3}$ : The folded leaf is a tool, the pleats of which increase the water-holding ability of the leaves. The multi-folded pith is not a tool, and the folds seem to have no containing function. So, Semliki wadges are discarded after one use, whereas Bossou's wadges may be re-used up to 122 times. At Semliki, only one species of plant is used for folding; at Bossou at least seven species are used. In making the wadge, at Semliki the raw material is folded over at its centre-point before being 'fed' into the mouth; at Bossou the raw material is stuffed directly into the mouth, starting at one end of the leaf. Most of these differences can be explained by the differing functions of the activities.

On the other hand, there are some similarities: Both procedures modify plant materials in order to fill up the buccal cavity, using folding as a technique to do so. The resulting pleats are evenly and similarly spaced, yielding a concertina-like artefact with alternating folds. The making of both types of artefact involves hand-mouth coordination. Most of these similarities can be explained by the similar biomechanics of the tasks.

Who cares if the number of folds in a wadge is odd or even? One answer is that this is the stuff of culture, not just of gross differences across populations, but also of nuanced variation, when cultural traits are basically similar but subtly different. Such trivial variants are common in human cultures, and the same may be true of nonhuman artefacts. This ethnographic note reminds us that culture is a layered phenomenon, and that if we operate on one level only, we may miss important features. Another answer is that researchers who work with artefacts, e.g. archaeologists, face challenges in inferring how those artefacts were produced. Etho-archaeology reminds us how difficult it can be to imagine the absent processes that result in material culture.

\section{ACKNOWLEDGEMENTS}

We thank: Uganda Wildlife Authority and Uganda National Council for Science and Technology for permission to work at Semliki, UWA rangers Alimosi Baluku, Patrick Biryomumsho, Charles Kasaija, Felix Anidraku, Justus Orobokiritto, and Elly Rutaro for assistance in the field; Moses Comeboy, Eriik Kasutama, Edson Katswamba, Jeremiah Nduthu for assistance in camp; Linda Marchant and Timothy Webster for research collaboration and comments on the manuscript; Lucie Salwiczek for graphics aid; Paco Bertolani for taking data at Kanyawara; an anonymous referee for helpful comments.

\section{REFERENCES}

1. Biro D, Sousa C, Matsuzawa T 2006. Ontogeny and cultural propagation of tool use by wild chimpanzees at Bossou, Guinea: Case studies in nut cracking and leaf folding. In: Cognitive Development in Chimpanzees. Matsuzawa $\mathrm{T}$, Tomonaga M, Tanaka M (eds), Springer, Tokyo, pp 476-508.

2. Sousa $C$ 2011. Use of leaves for drinking water. In: The Chimpanzees of Bossou and Nimba. Matsuzawa T, Humle T, Sugiyama Y (eds), Springer, Tokyo, pp 85-96.

3. Tonooka R 2001. Leaf-folding behavior for drinking water by wild chimpanzees (Pan troglodytes verus) at Bossou, Guinea. Anim Cogn 4:325-334.

4. Tonooka R, Inoue N, Matsuzawa T 1994. [Leaf-folding behavior for drinking water by wild chimpanzees at Bossou, Guinea: A field experiment and leaf selectivity.] Primate Res 10:307-313. [Japanese, with English summary]

5. Goodall J 1986. The Chimpanzees of Gombe: Patterns of
Behavior. Harvard University Press, Cambridge, MA.

6. Hunt KD, McGrew WC 2002. Chimpanzees in the dry habitats of Assirik, Senegal and Semliki Wildlife Reserve, Uganda. In: Behavioural Diversity in Chimpanzees and Bonobos. Boesch C, Hohmann G, Marchant LF (eds), Cambridge University Press, Cambridge, pp 35-51.

\section{<NOTE> \\ Preliminary Observations of Hand-Clasp Grooming by Chimpanzees at Bulindi, Uganda}

\author{
Matthew R. McLennan \\ Anthropology Centre for Conservation, Environment \& \\ Development, Oxford Brookes University, UK \\ (E-mail:mmclennan@brookes.ac.uk)
}

\section{INTRODUCTION}

The grooming hand-clasp (GHC) was the first documented social custom in wild chimpanzees ${ }^{1}$. It occurs when two chimpanzees seated opposite one another clasp hands overhead and groom each other's underarm with their free hand. The resulting configuration is strikingly symmetrical. Variant forms include one participant grasping their partner's hand or wrist, and 'wristto-wrist' in which participants rest their wrists against each other's forearm, usually one partner supporting most of the weight of both ${ }^{2,3}$. The behavior may originate from branch-clasp grooming - a universal behavior in chimpanzees ${ }^{4}$-in which participants grasp an overhead branch whilst grooming socially ${ }^{1}$. GHC has a patchy distribution across Africa. It occurs in more study communities than not (whether present, habitual or customary), yet is notably absent at three long-term sites: Bossou (Guinea), Gombe (Tanzania), and Budongo (Uganda) ${ }^{4,5}$. Records from new study sites are valuable because they increase our understanding of chimpanzee behavioral variation. Here I report preliminary observations of GHC in chimpanzees at Bulindi, Uganda.

\section{STUDY SITE}

Bulindi is a forest-agriculture ecotone, $25-\mathrm{km}$ south

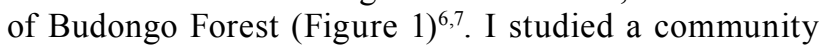
of $\geq 25$ chimpanzees for 18 months during 2006-2008. Although chimpanzees were unhabituated to close observation, from mid-2007 the community's six adult males showed signs of semi-habituation? ${ }^{7}$. Consequently, observations of social behavior including grooming and dominance interactions increased as the study progressed.

\section{OBSERVATIONS}

Two instances of GHC were observed in which the identity of participants was confirmed. At 0736 on 29 May 2007 we followed chimpanzee vocalizations to a clearing in heavily logged forest. Four adult males were seated on a rotting $\log , 27 \mathrm{~m}$ distant. Two males immediately climbed down behind the log while two (JL and MR) remained in view, staring with hair erect. Previous short-range encounters with chimpanzees generated alarm 


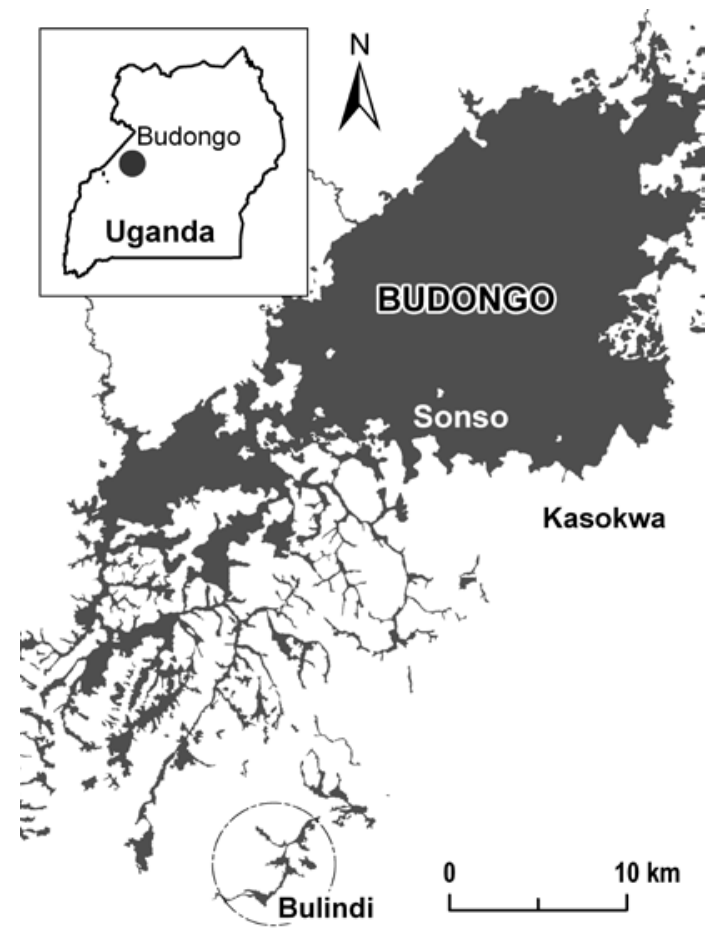

Fig.1. Map showing the location of Bulindi (at bottom) in relation to Budongo Forest in western Uganda. Gray areas represent forest. The approximate home range of Bulindi chimpanzees is encircled. The locations of the Sonso community in Budongo and nearby Kasokwa community (where grooming hand-clasp has not been seen) are also indicated. An unknown number of small chimpanzee groups utilize forest patches to the north and west of Bulindi.

and agitation, usually accompanied by threats from adult males ${ }^{7}$. However, on this occasion the males did not threaten us. After approximately two minutes, JL and MR began self-grooming while monitoring us; a third male $(\mathrm{JK})$ was peeping from behind the log. At $0808 \mathrm{KT}$ climbed on the $\log$ and stood glaring at us with erect hair. When he sat JL began grooming him; after several minutes they groomed mutually. At 0818 they raised their right arms overhead and performed the wrist-to-wrist variant of GHC (Figure 2a). Video analysis indicated that JL initiated or facilitated the bout by momentarily taking hold of KT's right forearm at elbow and wrist and gently pushing upwards (Figure 2b). Once extended, KT's wrist appeared supported by JL's wrist (Figure $2 \mathrm{a}$ ). The bout lasted 49 seconds (Video 1, available online at mahale.main.jp/ PAN/18 2/18(2) 03.html).

The second instance occurred on 31 July 2007. At 0859 we observed JL, KT and MR in a Parkia filicoidea tree, $55 \mathrm{~m}$ distant. JL and KT were grooming mutually. At 0904 they raised their left arms and performed wrist-to-wrist GHC for approximately 60 seconds. On this occasion JL rested his left hand/wrist on KT's forearm.

Two further instances of GHC were recorded. On both occasions grooming chimpanzees were observed in tree crowns from $\geq 80 \mathrm{~m}$ distance and identity of participants, and the form of GHC, was unconfirmed. In one case JL and KT formed a grooming clique with an estrous female but it was unclear if the two males performed GHC or if one performed it with the female. In the second case two adult males partially obscured by foliage performed GHC. Two other males in the party were identifiable (SL and MR). When the party moved off four adult males were present; the two unidentified males were almost certainly JL and KT.

Overhead branch-clasp grooming was witnessed three times: once it occurred between JL and KT, once between $\mathrm{KT}$ and SL, and once between an unidentified adult male and estrous female.

\section{DISCUSSION}

GHC is performed usually, but not exclusively, by adults ${ }^{1,5,8}$. While a possible male bias was indicated in some studies ${ }^{2,9}$, adults of both sexes participate in this activity $^{1,5}$. However, particular individuals ${ }^{9,10}$ and dyads $\mathrm{s}^{5,10}$ may perform GHC more often than other group members. Adult females at Bulindi were shy of human observers and most grooming observations involved adult males. Thus, further study is needed to establish the occurrence of GHC in females at this site. Two well-observed GHC bouts involved the same two prime adult males (JL and KT) who were also the likely participants in two additional instances. These males performed GHC twice during four well-observed bouts of mutual grooming totalling $157 \mathrm{~min}$. A third prime male (SL) groomed with KT during three bouts (118 min), but they did not perform GHC. $\mathrm{JL}$ and SL seldom interacted and were not seen grooming. Two young adult males (MR and JK) and an elderly male

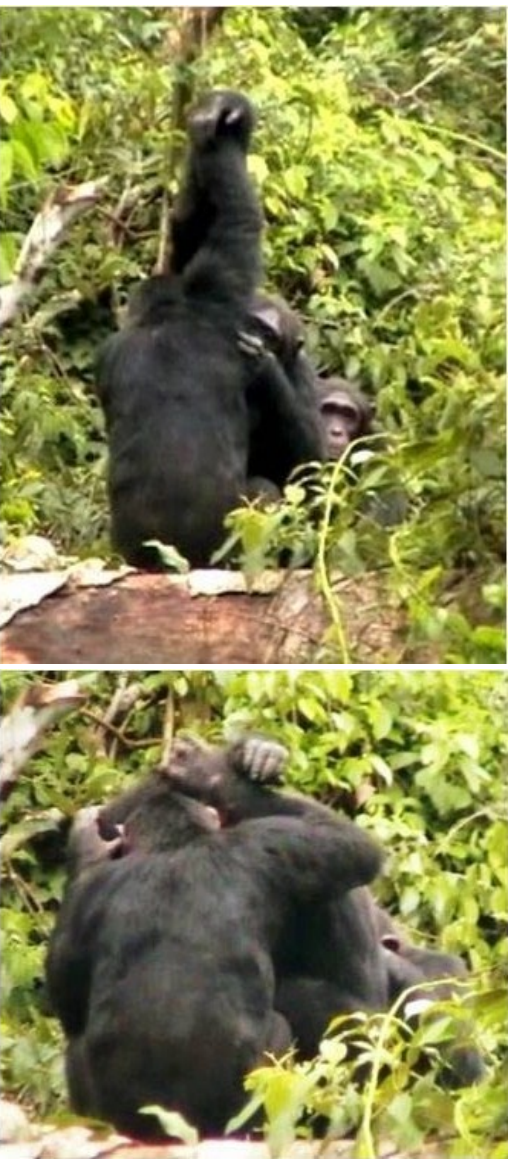

Figure 2a. Adult males Julius (JL) and Keeta (KT) performing grooming hand-clasp (May 2007); behind them a young adult male Murray (MR) is watching observers. $\mathrm{JL}$ and $\mathrm{KT}$ were the topranked males at Bulindi. Although in this videostill KT (facing camera) appears to rest his wrist on JL's wrist, possibly indicating his dominance (cf. reference 2), subsequent observations of social behavior suggested JL was alpha male (unpubl. data).

Figure 2b. Video-still showing JL (with back to camera) apparently initiating grooming handclasp by momentarily holding KT's forearm with both hands and gently pushing upwards. 
(LR) were also encountered regularly but were rarely seen grooming with other males. These preliminary observations suggest JL and KT-who both received submissive behavior from other males, including the largest male SL - had a GHC "partnership" ${ }^{10}$. More data are evidently needed, but we may tentatively classify GHC as 'habitual' at Bulindi ${ }^{4}$.

While GHC occurs at Bulindi, it has not been seen $25 \mathrm{~km}$ north in the Sonso community at Budongo ${ }^{11}$. GHC has not previously been reported present in one community yet absent in another so nearby (Mahale, where it occurs, and Gombe, where it does not, are separated by $150 \mathrm{~km}$; Kibale, where it occurs, and Sonso, where it does not, are $170 \mathrm{~km}$ apart). GHC has also not been seen in the small Kasokwa community bordering the southern edge of Budongo (Janette Wallis, pers. comm.) (see Figure 1). Bulindi chimpanzees use tools to excavate subterranean bee nests for honey - a behavior not recorded at Sonso or elsewhere in western Uganda ${ }^{12}$. The occurrence of GHC at Bulindi provides further evidence of behavioral variation among chimpanzees in the Budongo region. Multiple small chimpanzee groups inhabit forests patches within the cultivated landscape south of Budongo ${ }^{13}$. Chimpanzees occur north of Bulindi, closer to Budongo's southern border (e.g. around Kasongoire Forest Reserve). Future studies should aim to establish the status of GHC among chimpanzees in this intervening area.

GHC was first identified in 5/8 (63\%) long-term study communities ${ }^{4}$. An expanded data-set reveals it occurs in 14/17 communities (82\%), including two Pan paniscus communities $^{5,8,14}$. If we include Bulindi and Kasokwa it occurs in 15/19 (79\%). This suggests GHC is usually present in wild populations. Nevertheless, it is easier to confirm presence than absence in unhabituated or semihabituated communities. Despite the emerging high prevalence of GHC in wild chimpanzees, the evidence for its absence at three sites (Bossou, Gombe, Sonso) is firmit has not been seen at these sites in decades of fieldwork. GHC has emerged spontaneously in one captive colony ${ }^{15}$ and possibly one sanctuary-released group ${ }^{9}$. This indicates $\mathrm{GHC}$ is a dynamic social custom that potentially emerges and disappears in local populations repeatedly over time.

\section{ACKNOWLEDGEMENTS}

I thank the President's Office, the Uganda National Council for Science and Technology, and Uganda Wildlife Authority for permission to work in Uganda. Dan Balemesa, Gerald Mayanda, Tom Sabiiti, Moses Ssemahunge and Jane Stokoe helped in the field. Research was funded by the ESRC and NERC, and the Leverhulme Trust (project reference: F/00 $382 / \mathrm{F}$ ). The manuscript was improved by comments from W.C. McGrew.

\section{REFERENCES}

1. McGrew WC, Tutin CEG 1978. Evidence for a social custom in wild chimpanzees? Man 13:234-251.

2. McGrew WC, Marchant LF, Scott SE, Tutin CEG 2001. Intergroup differences in a social custom of wild chimpanzees: The grooming hand-clasp of the Mahale Mountains. Curr Anthropol 42:148-153.

3. Nakamura M, Uehara S 2004. Proximate factors of different types of grooming hand-clasp in Mahale chimpanzees: Implications for chimpanzee social customs. Curr Anthropol 45:108-114.
4. Whiten A, Goodall J, McGrew WC, Nishida T, Reynolds V, Sugiyama Y, Tutin CEG, Wrangham RW, Boesch C 2001. Charting cultural variation in chimpanzees. Behaviour 138:1481-1516.

5. Nakamura M 2002. Grooming-hand-clasp in Mahale M Group chimpanzees: Implications for culture in social behaviours. In: Behavioural Diversity in Chimpanzees and Bonobos. Boesch C, Hohmann G, Marchant LF (eds), Cambridge University Press, Cambridge, pp. 71-83.

6. McLennan MR 2010. Case study of an unusual humanchimpanzee conflict at Bulindi, Uganda. Pan Afr News 17:1-4.

7. McLennan MR, Hill CM 2010. Chimpanzee responses to researchers in a disturbed forest-farm mosaic at Bulindi, western Uganda. Am J Primatol 72:907-908.

8. Webster TH, Hodson PR, Hunt KD 2009. Grooming handclasp by chimpanzees of the Mugiri community, ToroSemliki Wildlife Reserve, Uganda. Pan Afr News 16:5-7.

9. Humle T, Colin C, Raballand E 2009. Preliminary report on hand-clasp grooming in sanctuary-released chimpanzees, Haut Niger National Park, Guinea. Pan Afr News 16:7-10.

10. Bonnie KE, de Waal FBM 2006. Affiliation promotes the transmission of a social custom: Hand-clasp grooming among captive chimpanzees. Primates 47:27-34.

11. Reynolds V 2005. The Chimpanzees of the Budongo Forest. Oxford University Press, Oxford.

12. McLennan MR 2011. Tool-use to obtain honey by chimpanzees at Bulindi: New record from Uganda. Primates 52:315-322.

13. McLennan MR 2008. Beleaguered chimpanzees in the agricultural district of Hoima, western Uganda. Primate Conserv 23:45-54.

14. Fruth BI, Hohmann G, Beuerlein MM, McGrew WC 2006. Grooming hand clasp by bonobos of Lui Kotal, Democratic Republic of Congo. Pan Afr News 13:6-8.

15. de Waal FBM, Seres M 1997. Propagation of handclasp grooming among captive chimpanzees. Am J Primatol 43:339-346.

\section{<NOTE>}

\section{Evaluating the Effectiveness of a 10-Year Old Great Ape Conservation Project in Cameroon}

\author{
Nikki Tagg ${ }^{1,2}$, Charles-Albert \\ Petre $^{3,4} \&$ Jacob Willie ${ }^{1}$ \\ 1 Projet Grands Singes, Cameroon, Cameroon. \\ 2 Centre for Research and Conservation, Royal Zoological \\ Society of Antwerp, Belgium. \\ 3 Liège University, Belgium. \\ 4 Gembloux Agro-Bio Tech, Belgium. \\ (E-mail: Nikki.tagg@kmda.org)
}

The world faces a massive human-accelerated biodiversity decline. Western lowland gorillas (Gorilla gorilla gorilla) and central chimpanzees (Pan troglodytes troglodytes) are heavily concerned by this decline, with a range-wide mean of $50 \%$ having perished ${ }^{1,2}$, due to hunting for meat, disease and habitat loss and disturbance ${ }^{3,4,5,6}$. A large majority (approximately 80\%) of all remaining populations of chimpanzees and gorillas live outside protected areas (PAs) ${ }^{7,8}$ where human pressures are high. A serious everyday threat to great ape survival is hunting for meat ${ }^{3}$ and as a result of the poverty of an ever-growing 